\title{
The impact of hyperactivity and leptin on recovery from anorexia nervosa
}

\author{
A. A. van Elburg ${ }^{1,3}$, M. J. H. Kas ${ }^{2}$, J. J. G. Hillebrand ${ }^{2,3}$, R. J. C. Eijkemans ${ }^{4,5}$, H. van Engeland ${ }^{1}$ \\ ${ }^{1}$ Department of Child and Adolescent Psychiatry, Rudolf Magnus Institute of Neuroscience, Utrecht, The Netherlands \\ ${ }^{2}$ Department of Pharmacology and Anatomy, University Medical Center Utrecht, Utrecht, The Netherlands \\ ${ }^{3}$ Rintveld Center for Eating Disorders, Altrecht Mental Health Institute, Zeist, The Netherlands \\ ${ }^{4}$ Department of Reproductive Medicine and Gynaecology, University Medical Center Utrecht, Utrecht, The Netherlands \\ ${ }^{5}$ Department of Public Health, Erasmus Medical Center, Rotterdam, The Netherlands
}

Received 27 October 2006; Accepted 12 April 2007; Published online 26 May 2007

(C) Springer-Verlag 2007

\begin{abstract}
Summary In anorexia nervosa (AN), hyperactivity is observed in about $80 \%$ of patients and has been associated with low leptin levels in the acute stage of AN and in anorexia animal models. To further understand the importance of this correlation in AN, we investigated the relationship between hypoleptinaemia and hyperactivity in AN patients longitudinally and assessed their predictive value for recovery.

Body weight, activity levels, and serum leptin levels were assessed in adolescents and adult AN patient groups at the start and during treatment, up to a year. In the adolescent group, initial leptin and activity levels were correlated. This negative correlation changes over time into a positive correlation with physiological recovery. Treatment outcome in both groups could be predicted by initial BMI and leptin levels but not by activity levels. No major relationship of activity with the course of recovery was detected, suggesting that in contrast to the acute stage of the disease, leptin and activity levels during the recovery process are dissociated.
\end{abstract}

Keywords: Anorexia nervosa; hyperactivity; leptin; recovery; eating disorders; physical activity

\section{Introduction}

Anorexia nervosa (AN) is a complex eating disorder of unknown etiology, which primarily affects adolescent girls and young women (Lucas et al., 1991, 1999; Hoek and Hoeken, 2003). It is characterized by aberrant patterns of eating behavior and weight regulation which result in weight loss and endocrine abnormalities such as amenorrhea, disturbances in attitude and perception about weight and shape,

Correspondence: Dr. Annemarie A. van Elburg, Rintveld Center for Eating Disorders, Altrecht Mental Health Institute, Oude Arnhemseweg 260, 3705 BK Zeist, The Netherlands

e-mail: a.van.elburg@altrecht.nl and an intense fear of gaining weight (American Psychiatric Association, 1994).

Other symptoms, such as perfectionism, obsessivecompulsive behavior, and social anxiety are observed in many but not all patients (Piran et al., 1985; Casper and Jabine, 1996). In addition, a proportion of AN patients (31-80\%, Hebebrand et al., 2003) displays abnormally high physical activity levels and overexercises. Almost all patients show a constant, agitated restlessness when they are emaciated but before they become lethargic in the final stages of starvation. The exact nature of hyperactivity in anorexia nervosa remains to be clarified. It appears that neurobiological factors and conscious attempts to burn calories in order to loose more weight coexist (Casper, 1998, 2006).

Animal models mimicking AN weight loss and hyperactivity, such as the activity based anorexia (ABA) model (Routtenberg and Kuznesof, 1967; Kas et al., 2003) or the semistarvation induced hyperactivity (SIH) model (Pirke, 1993; Exner et al., 2000) have been helpful in the search for a possible biological drive or changes in physiological parameters that trigger food restriction and hyperactivity. Studies using these preclinical models showed that anorectic rats (Kas et al., 2003) and mice (Gelegen et al., 2007) have reduced plasma leptin levels and that treating food restricted rats with leptin suppresses the development of hyperactivity (Exner et al., 2000; Hillebrand et al., 2005a).

Leptin is an adipocyte secreted hormone, product of the ob gene (Zhang et al., 1994), which plays a pivotal role in starvation situations with regard to energy homeostasis as 
well as other physiological processes, including reproductive functioning (Cioffi et al., 1996; Moschos et al., 2002). Plasma leptin levels rapidly decrease during weight loss (Ahima et al., 1996) and are extremely low in AN patients (Calandra et al., 2003). However, leptin levels in recently weight recovered patients have been found to be higher than in BMI-matched controls, and are thought to contribute to difficulties with further weight restoration and maintenance (Eckert et al., 1998; Holtkamp et al., 2004; Mantzoros et al., 2006). Holtkamp et al. have shown that patients leptin levels are negatively correlated with motor restlessness scores (2003) and with physical activity in acutely ill AN patients (2006). Thus, pre-clinical and clinical observations suggest that hypoleptinemia is an important factor underlying excessive physical activity in AN. The meaning of these findings for recovery have however not been studied in detail to date.

The objective of this study was to investigate the relationship between hyperactivity, serum leptin levels, and recovery in AN patients.

\section{Materials and methods}

\section{Subjects}

Patients were included in the study after they and/or their parents gave informed consent. The sample was recruited in two specialized eating disorder treatment centers (clinics), one for adolescents 12-17 years (the University Medical Center Utrecht [UMCU], 31 subjects) and one for adults older than 17 years (Rintveld, 30 subjects). The clinical research protocol for this prospectively designed, cohort follow-up study was approved by the UMCU Ethics Review Committee.

Inclusion criteria were: (1) AN diagnosed according to the DSM-IV (American Psychiatric Association, 1994) on the basis of a structured interview using the Eating Disorders Examination (Cooper et al., 1989); (2) comorbidity restricted to depression or anxiety disorders; (3) secondary amenorrhea; (4) no use of steroid contraceptives; (5) no clinical history of concurrent illnesses. Sixty-one female patients were included, 24 of the AN purging type, 37 of the AN restricting type.

\section{Study design}

After initial psychiatric assessment, patients entered a structured treatment program aimed at restoration of the patient's weight, normalization of eating patterns, body image, anorectic cognitions and family and social functioning. Weight gain was targeted at $0.5-1.0 \mathrm{~kg} /$ week in accordance with clinical guidelines. Weight recovery was defined as a weight within the normal range for age $(>\mathrm{SD}-1.5$ corresponding with a body mass index [BMI] of approximately $19 \mathrm{~kg} / \mathrm{m}^{2}$ for adults) and target weight as the weight at which patients resumed a regular menstrual cycle, defined as three menstrual periods at three to five week intervals. Individual, group and family therapy techniques were used to change the patients' aberrant body perception and cognition.

Data was collected from patient acceptance into the treatment program, in the acute stage of the illness, until the end point, defined as recovery (return of regular menses), or up to one year after begin of treatment.

Body weight was measured once a week. The degree of underweight was calculated using BMI $\left(\mathrm{kg} / \mathrm{m}^{2}\right)$ and BMI computed into $\mathrm{z}$ scores which describe the distance in SD from the mean BMI for that age (using a software program provided by the Netherlands Organization for Applied Scientific Research to account for differences in age) and to relate the data to Dutch population references (Van Buuren and Fredriks, 2001).

Blood samples for hormonal analysis were obtained by venepuncture at admission and every two weeks thereafter at $4 \mathrm{pm}$, up to the maximum study period of one year.

\section{Activity measures}

Physical activity levels were measured by trained nurses once a week. In an earlier study using actometer measurement as gold standard we established the reliability and validity of nurse observations of patient activity (Van Elburg et al., 2007). Nurses were instructed to observe activity, defined as the amount of motor restlessness (inability to sit still or moving arms or legs while seated, walking through the ward without reason), abnormal motor activity, and excessive exercise, and to score their observations on a visual analogue scale $(0-10)$.

\section{Leptin assay}

Plasma samples were stored at $-80^{\circ} \mathrm{C}$ prior to determination. Leptin was measured using a sensitive RIA (Human-Leptin-RIA sensitive, Mediagnost, Tubingen, Germany), intra-assay CV of $5 \%$, inter-assay CV of $7.6 \%$.

\section{Statistics}

At the study endpoint, follow-up data was collected on the basis of which, patients were divided into three groups according to their recovery stage: no weight recovery (NWR), weight recovery without resumption of menses (WR) and weight and cycle recovery (WCR). Data are presented as the mean \pm SD if distributed normally, otherwise as the median and range. Kruskal-Wallis tests were used if data were not normally distributed. In case data were normally distributed ANOVA was used. Linear multiple regression analysis (backward elimination) was also carried out to predict physical activity using BMI z scores and $\log 10$ leptin levels as predictor variables. A $p$-value of $<0.05$ was considered to be statistically significant.

To determine the associations of weight, leptin and physical activity levels with the rate of recovery as defined by the menstrual cycle at end point, univariate and multivariate Cox proportional hazards analyses were performed. This method of analysis estimates a linear regression model of parameters against the logarithm of the hazard (or instantaneous risk) of the menstrual cycle recovery during follow-up. Initial values of parameters were used as potential predictors. Parameters with univariate $p<0.30$ were candidate predictors for the multivariate model; backward elimination of parameters (with $p<0.05$ for inclusion) was used to determine the set of most predictive parameters. Because of potential differences in prognosis averages between the two treatment centers, the analysis was stratified by clinic. The analysis was first performed with initial values of weight, leptin and physical activity levels as predictors and subsequently with longitudinal values, using Cox regression with time-dependent covariates. A further analysis of longitudinal data focused on the evolvement of the association between leptin levels and activity levels during the course of treatment. A linear mixed model was used, predicting physical activity from log 10 leptin levels, follow-up time measured in weeks and final recovery status as predictor variables. A potential difference between the recovered and nonrecovered group in the evolvement of the association between leptin levels and activity levels over time was tested by an interaction of (log 10 leptin) ${ }^{*}$ week $^{*}$ group.

Statistical analysis was performed using SPSS for Windows (release 11.5, SPSS Inc., Chicago, IL) and S-plus software (MathSoft Inc., Seattle, WA, version 2000). 
Table 1. Demographics (mean $\pm S D$ ) of 61 young women diagnosed with anorexia nervosa. Data are presented for the entire group, and separately for women presenting with no weight recovery (NWR), with weight recovery (WR) only and with weight \pm cycle recovery (WCR) during the study period of maximum 12 months

\begin{tabular}{|c|c|c|c|c|}
\hline Variables & Total group $(n=61)$ & NWR $(n=19)$ & $\mathrm{WR}(n=18)$ & WCR $(n=24)$ \\
\hline Age (years) & $18.2 \pm 3.1$ & $17.3 \pm 2.2$ & $16.9 \pm 3.2$ & $19.8 \pm 3.0^{*}$ vs. NWR $+\mathrm{WR}$ \\
\hline Weeks in study & $33.5 \pm 11.4$ & $32.8 \pm 11.6$ & $36.9 \pm 14.0$ & $31.6 \pm 8.8$ \\
\hline Bodyweight, initial z scores & $-3.8 \pm 1.6$ & $-4.2 \pm 1.9$ & $-3.2 \pm 1.5$ & $-3.8 \pm 1.2$ \\
\hline Bodyweight end of study & $-1.3 \pm 1.1$ & $-2.1 \pm 0.8^{*}$ vs. WCR $+\mathrm{WR}$ & $-0.9 \pm 0.5$ & $-0.9 \pm 0.6$ \\
\hline Amenorrhea (months) & $24.4 \pm 22.8$ & $20.5 \pm 13.5$ & $30.0 \pm 30.5$ & $23.9 \pm 23.6$ \\
\hline Initial leptin $(\mu \mathrm{g} / 1)$ & $2.1(0.5-13.3)$ & $2.9(0.7-8.6)$ & $1.5(0.5-4.6)$ & $2.4(0.7-13.3)$ \\
\hline AN restrictive type $(\%)$ & 69 & 79 & 72 & 58 \\
\hline Neuroleptics $(\%)$ & 57 & 47 & 55 & 58 \\
\hline SSRIs (\%) & 33 & 26 & 22 & 50 \\
\hline Initial activity score & $52.5 \pm 26.9$ & $49.1 \pm 27.9$ & $56.2 \pm 24.9$ & $52.4 \pm 28.8$ \\
\hline
\end{tabular}

* $p<0.01$

\section{Results}

The mean age of the whole patient group was $18.2 \pm 3.1$ (SD) years, $15.9 \pm 1.2$ years for the adolescent group and $20.6 \pm 2.9$ years for the adult group. Average BMI at admission was $15.4 \pm 1.3 \mathrm{~kg} / \mathrm{m}^{2}$, which corresponds to a BMI $\mathrm{z}$ score of $-3.8 \pm-1.6$ (Table 1). Within the one year time frame of the study, $42(69 \%)$ patients met the weight recovery objectives. Of them 24 (39\% of the total, $57 \%$ of the weight recovered group) also recovered their menstrual cycles; 18 patients recovered in weight without the resumption of a menstrual cycle. Nineteen patients (31\%) remained at a low weight throughout the year.

There were no significant differences among the three outcome groups in initial body weight, duration of illness (defined by duration of amenorrhea), duration of study participation, type of AN (restrictive or purging type), plasma leptin levels, nurse ratings of physical activity levels, amount of prescribed neuroleptics, or SSRIs (Table 1). Fourteen patients used olanzapine, 7 in each group (clinic) with dosages ranging from 5 to $15 \mathrm{mg}$ /day, average dose $7.5 \mathrm{mg}$ in both clinics. A difference in age was observed. The group that recovered in weight and resumed a menstrual cycle was significantly older than the non-recovered group, which result also corresponds with the clinic where they were treated. Rates of recovering weight and menstrual cycle differed in the two clinics: $16 \%$ of the patients in the adolescent group recovered versus $63 \%$ of the patients in the adult group.

For the group as a whole, initial leptin levels did not correlate with nurse ratings of activity, the scatter plot showing a U-shape $(p=0.082)$. After stratification by clinic and adjusted for BMI z sore, the adolescent patient group showed a linear association between activity levels and $\log 10$ serum leptin levels (partial correlation: $-0.40, p=0.027$ ), whereas the adult group shows the U-shaped scatter plot, $p=0.12$ (Fig. 1).

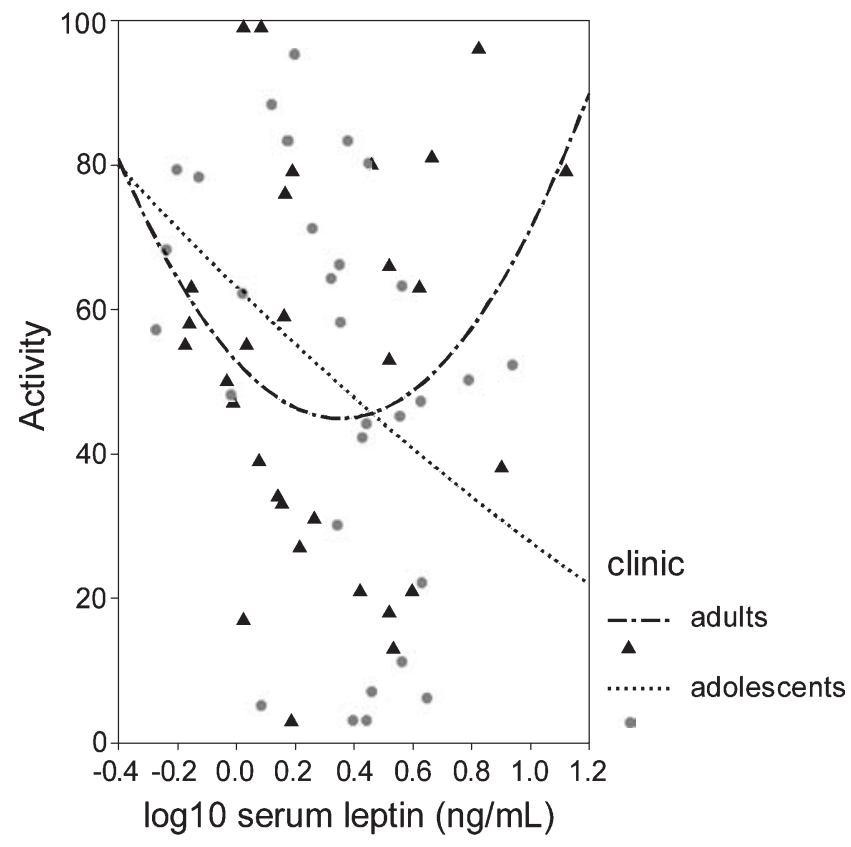

Fig. 1. Scatter plot of initial $\log 10$ serum leptin levels versus observed levels of levels of activity in two groups of patients with AN. Activity is defined as the amount of motor restlessness, abnormal motor activity and excessive exercise, as observed by nurses. $p=0.027$ for the adolescent group $(N=31), p=0.12$ for the adult group $(N=30)$

Next, we analyzed the predictive value of initial BMI $\mathrm{z}$ score, activity and plasma leptin levels on complete (weight and cycle) recovery. In a univariate analysis, initial leptin levels and initial BMI z scores predicted a favourable outcome, whereas initial activity levels showed no correlation. Multivariate analysis showed that leptin was the sole significant variable (Table 2).

Analysis of the longitudinal data using a Cox model with time-dependent covariates showed leptin and BMI z score as main influences on recovery of menstrual cycle. Adding 
Table 2. Association of initial BMI z scores, leptin, and activity levels with time to recovery of menstrual cycle

\begin{tabular}{lccl}
\hline Parameter & $\begin{array}{l}\text { Univariate } \\
p \text { value }\end{array}$ & $\begin{array}{l}\text { Multivariate } \\
p \text { value }\end{array}$ & $\begin{array}{l}\text { Hazard ratio* } \\
(95 \% \mathrm{CI})\end{array}$ \\
\hline BMI z score & 0.0014 & 0.15 & \\
Leptin level & $<0.001$ & $<0.001$ & $1.97(1.31-2.97)$ \\
Activity level & 0.84 & 0.96 & \\
\hline
\end{tabular}

* The change in hazard when the parameter is increased by one standard deviation

Cox regression stratified by treatment center

Table 3. Time-dependent analysis of the association of BMI $z$ scores, leptin and activity levels with recovery of menstrual cycle

\begin{tabular}{lcll}
\hline Parameter & $\begin{array}{l}\text { Univariate } \\
p \text { value }\end{array}$ & $\begin{array}{l}\text { Multivariate } \\
p \text { value }\end{array}$ & $\begin{array}{l}\text { Hazard ratio* } \\
(95 \% \mathrm{CI})\end{array}$ \\
\hline BMI z score & $<0.001$ & 0.003 & $2.1(1.3-3.3)$ \\
Leptin & $<0.001$ & 0.022 & $1.12(1.02-1.24)$ \\
Activity & 0.9 & & \\
\hline
\end{tabular}

* Hazard ratio for standardised parameters, with $95 \%$ confidence interval. The hazard ratio represents the change in hazard when the parameter is increased by one standard deviation

Cox regression stratified by clinic

activity did not improve prediction in the model (Table 3 ). The linear mixed model analysis showed that the recovered (WCR) and non-recovered patients (WR and NWR) differed significantly in the way the relationship between leptin and physical activity changed during the treatment period $(P=0.016$, test for interaction): the correlation between leptin and physical activity remained the same during treatment $(r=-0.28)$ in the NWR and WR groups, whereas in the WCR group it changed from an initial value of $r=-0.22$ at 16 weeks to $r=+0.58$ at 52 weeks.

\section{Discussion}

In this longitudinal study we measured body weight, plasma leptin levels, and activity levels in a clinical sample of AN patients during recovery.

We replicated the finding from animal and human studies (Kas et al., 2003; Holtkamp et al., 2003, 2006) that initial $\log 10$ leptin levels and activity levels in the acute stage of the illness in a young patient population are correlated, and found a linear correlation in our adolescent age group. In our adult group we found a $\mathrm{U}$ shaped correlation between activity and plasma leptin levels, which suggests that hyperactivity is age-related. Over time however, physical activity did not predict outcome and no major relationship between physical activity levels and the course of recovery could be detected for neither the adolescent nor the adult group.
Contrary to Holtkamp (2003) we rated activity during treatment and observed by nurses instead of relying on anamnestic reports of physical activity in the months before admission or during the acute stage of the illness. In an earlier study (Van Elburg et al., 2007) we showed that nurses can reliably rate physical activity levels of anorexia nervosa patients. Measurements of a patient's physical activity levels by nurses correlate with activity scores from actometers, the gold standard reference. Patient self-reports did not correlate with the actometer scores. In clinical practice a variety of methods has been used to evaluate hyperactivity, but based on our previous findings conclusions drawn from studies using self-report or expert report not based on observation should be considered only with caution.

Once patients were divided into different outcome groups at the end of the study period differences in levels of physical activity and leptin were found, even though initial levels were not significantly different. Initial plasma leptin levels for all groups were clearly below normal values. Only the WCR-group reached average levels in the normal range at the end of the study period. WR patients clearly responded to treatment and recovered in weight, however, their subnormal leptin levels most probably did not permit them to recover at the level of the gonadal axis.

Several studies (Haupt et al., 2005; Wang et al., 2006) have discussed the relationship between leptin and the use of atypical antipsychotics such as olanzapine, so far only in normal weight schizophrenic or psychotic patients. The results show that olanzapine in the first weeks of treatment may cause a surge in circulating leptin (Wang et al., 2006) but that elevated plasma leptin levels in chronically treated patients with schizophrenia are strongly predicted by adiposity, similar to untreated healthy individuals (Haupt et al., 2005).

In a previous study (Hillebrand, 2005b), we demonstrated that olanzapine reduces activity levels in AN patients without affecting body weight and plasma leptin levels. In our current study, $57 \%$ of patients used antipsychotics (neuroleptics), which might have influenced the relationship between leptin and activity scores. However, Cox regression analysis with medication as predictor showed no correlation (univariate: neuroleptics: $p=0.6$, SSRIs: $p=0.3$ ). Holtkamp et al. (2003, 2006) unfortunately do not report use of neuroleptics in their study populations.

In summary, our study replicates earlier findings in both rodents and humans that hypoleptinaemia and high physical activity levels are related in acutely ill AN patients of a young age, but we did not find the same relationship in an older patient group. The negative correlation between leptin and activity that exists in the acute phase of the illness 
changes over the course of 16 weeks to become a positive correlation in the second half of the treatment period, as a result of recovery. In the non recovered patients the negative correlation continues. Furthermore, our data indicate that plasma leptin level is a good predictor of recovery in AN, however, we could not detect any major relationship between physical activity levels and the course of recovery. The latter indicates that leptin and physical activity levels during the recovery process are, in contrast to the acute stage of the disease, dissociated. Further detailed assessment of this relationship is required to elaborate on these findings and their implications for clinical practice.

\section{Acknowledgements}

The authors wish to express their gratitude to the research assistants Maartje Snoek and Hanneke Hop for their help with the data collection. Claudia Mika is gratefully acknowledged for assistance with the radioimmunoassay.

\section{References}

Ahima RS, Prabakaran D, Mantzoros C, Qu D, Lowell B, Maratos-Flier E, Flier JS (1996) Role of leptin in the neuroendocrine response to fasting. Nature 382: 250-252

American Psychiatric Association (1994) Diagnostic and statistical manual of mental disorder, 4th edn. American Psychiatric Association, Washington DC

Calandra C, Musso F, Musso R (2003) The role of leptin in the etiopathogenesis of anorexia nervosa and bulimia. Eat Weight Disord 8 : $130-137$

Casper R (1998) Behavioral activation and lack of concern, core symptoms of anorexia nervosa? Int J Eat Disord 24: 381-393

Casper R (2006) The 'drive for activity' and "restlessness" in anorexia nervosa: potential pathways. J Affect Disord 92: 99-107

Casper R, Jabine L (1996) An eight-year follow-up: outcome from adolescent compared to adult onset anorexia nervosa. J Youth Adolesc 25: 499-517

Eckert ED, Pomeroy C, Raymond N, Kohler PF, Thuras P, Bowers CY (1998) Leptin in anorexia nervosa. J Clin Endocrinol Metabolism 83: 791-795

Exner C, Hebebrand J, Remschmidt H, Wewetzer C, Ziegler A, Herpertz S, et al (2000) Leptin suppresses semi-starvation induced hyperactivity in rats: implications for anorexia nervosa. Mol Psychiatry 5: 476-481

Gelegen C, Collier DA, Campbell IC, Oppelaar H, van den Heuvel J, Adan RA et al (2007) Difference in susceptibility to activity-based anorexia in two inbred strains of mice. Eur Neuropsychopharmacol 17(3): 199-205

Harrell FE Jr, Lee KL, Mark DB (1996) Multivariable prognostic models: issues in developing models, evaluating assumptions and adequacy, and measuring and reducing errors. Statist Med 15: 361-387

Haupt DW, Luber A, Maeda J, Melson AK, Schweiger JA, Newcomer JW (2005) Plasma leptin and adiposity during antipsychotic treatment of schizophrenia. Neuropsychopharmacology 30: 184-191
Hebebrand J, Exner C, Hebebrand K, Holtkamp K, Casper RC, Remschmidt H, Herpertz-Dahlmann B, Klingenspor M (2003) Hyperactivity in patients with anorexia nervosa and in semistarved rats: evidence for a pivotal role of hypoleptinemia. Psychol Behav 79: $25-37$

Hillebrand JJG, Koeners MP, de Rijke CE, Kas MJ, Adan RA (2005a) Leptin treatment in activity-based anorexia. Biol Psych 58: 165-171

Hillebrand JJG, van Elburg AA, Kas MJ, van Engeland H, Adan RA (2005b) Olanzapine reduces physical activity in rats exposed to activity-based anorexia: possible implications for treatment of anorexia nervosa? Biol Psych 58: 651-657

Hoek HW, van Hoeken D (2003) Review of the prevalence and incidence of eating disorders. Int J Eat Disord 34: 383-396

Holtkamp K, Herpertz-Dahlmann B, Mika C, Heer M, Heussen N, Fichter MM, Herpertz S, Senf W, Blum WF, Schweiger U, Warnke A, Ballauff A, Remschmidt H, Hebebrand J (2003) Elevated physical activity and low leptin levels co-occur in patients with anorexia nervosa. J Clin Endocrinol Metabolism 88: 5169-5174

Holtkamp K, Hebebrand J, Mika C, Heer M, Heussen N, HerpertzDahlmann B (2004) High serum leptin levels subsequent to weight gain predict renewed weight loss in patients with anorexia nervosa. Psychoneuroendocrinology 29: 791-797

Holtkamp K, Herpertz-Dahlmann B, Hebebrand K, Mika C, Kratsch J, Hebebrand J (2006) Physical activity and restlessness correlate with leptin levels in patients with adolescent anorexia nervosa. Biol Psychiatry 60(3): 311-313

Kas MJ, van Elburg AA, van Engeland H, Adan RA (2003) Refinement of behavioural traits in animals for the genetic dissection of eating disorders. Eur J Pharmacol 480: 13-20

Lucas AR, Beard CM, O'Fallon WM, Kurland LT (1991) 50-Year trends in the incidence of anorexia nervosa in Rochester, Minn.: a populationbased study. Am J Psychiatry 148: 917-922

Lucas AR, Crowson CS, O'Fallon WM, Melton LJ 3rd (1999) The ups and downs of anorexia nervosa. Int J Eat Disord 26: 397-405

Mantzoros C, Flier JS, Lesem MD, Brewerton TD, Jimerson DC (2006) Cerebrospinal fluid leptin in anorexia nervosa: correlation with nutritional status and potential role in resistance to weight gain. J Clin Endocrinol Metabolism 82: 1845-1851

Moschos S, Chan JL, Mantzoros C (2002) Leptin and reproduction: a review. Fert Steril 77: 433-444

Piran N, Kennedy S, Garfinkel P, Owens M (1985) Affective disturbances in eating disorders. J Nervous Mental Dis 173: 395-400

Pirke KM, Broocks A, Wilckens T, Marquard R, Sweiger U (1993) Starvation-induced hyperactivity in the rat: the role of endocrine and neurotransmitter changes. Neurosci Biobehav Rev 17: 287-294

Routtenberg A, Kuznesof A (1967) Self-starvation of rats living in activity wheels on a restricted feeding schedule. Psychol Behav 42: $156-163$

Van Buuren S, Fredriks M (2001) Worm plot: a simple diagnostic device for modelling growth reference curves. Stat Med 30: 1259-1277

Van Elburg AA, Hoek HW, Kas MJH, van Engeland H (2007) Nurse evaluation of hyperactivity in anorexia nervosa: a comparative study. Eur Eat Disord (Review in press)

Wang HC, Chen PS, Lee IH, Yang YK, Yeh TL, Lu RB (2006) Rapid leptin elevation after initiation of olanzapine? Neuropsychobiology 54: $182-185$

Zhang Y, Proenca P, Maffei M, Barone M, Leopold L, Friedman JM (1994) Positional cloning of the mouse obese gene and its human homologue. Nature 372: 425-432 